\title{
DESENVOLVIMENTO DE UM SISTEMA PARA GERENCIAMENTO PLANIMÉTRICO E TOPOGRÁFICO DE ÁREAS AGRÍCOLAS: WEB CALC-GEO
}

\author{
Vinício Fragoso Mendes ${ }^{1}$ \\ Thiago Vieira de Souza Eleutério² \\ Melissa Heringer Silveira ${ }^{3}$ \\ Pedro David Netto Silveira ${ }^{4}$
}

Resumo: Os dados geram informações, e com o tratamento de dados geográficos, pode-se obter informações topográficas. Objetivou-se com este trabalho, o desenvolvimento e apresentação de uma aplicação web, denominada Web Calc-Geo, capaz de realizar cálculos planimétricos. O sistema foi desenvolvido por um estudo sobre coletas e análises de dados, utilizando as linguagens de programação PHP e JavaScript, de marcação HTML e estilização CSS. Foi realizado um teste, onde foram coletados dados geográficos e inseridos manualmente na aplicação. Com os dados no sistema, foi possível visualizar os resultados automaticamente, além da geração do croqui e de um arquivo gpx, utilizado pelo programa de computador Google Earth, para uma visualização real da área em um mapa. Os dados do sistema podem ser vistos em qualquer navegador web, e além disto, mediante a análise, demonstrou ser atraente para utilização. Os usuários devem se ater a precisão dos dados que irá inserir no sistema, para não haver descontentamento por resultados incoerentes. Os resultados encontrados permitem afirmar que o sistema tem potencial para utilização.

Palavras-chave: GPS; Tecnologia; WebSite.

\footnotetext{
${ }^{1}$ Tecnologia em Análise e Desenvolvimento de Sistemas/Instituto Federal do Espírito Santo - Campus de Alegre, Brasil. E-mail: vinicio0408@gmail.com.

${ }^{2}$ Tecnologia em Análise e Desenvolvimento de Sistemas/Instituto Federal do Espírito Santo - Campus de Alegre, Brasil. E-mail: thiagoamamuitojesus@hotmail.com.

${ }^{3}$ Tecnologia em Análise e Desenvolvimento de Sistemas/Instituto Federal do Espírito Santo - Campus de Alegre, Brasil. E-mail: melissa.heringer@gmail.com.

${ }^{4}$ Tecnologia em Análise e Desenvolvimento de Sistemas/Instituto Federal do Espírito Santo - Campus de Alegre, Brasil. E-mail: pedro.silveira@ifes.edu.br.
} 